\title{
Ciclo térmico para la obtención de una ferrita de Ni-Zn: (I) Diseño de la etapa de sinterización
}

\author{
A. BARBA, C. CLAUSELL, M. MONZÓ, J.C JARQUE \\ Instituto de Tecnología Cerámica. Universitat Jaume I. Castellón. Spain.
}

\begin{abstract}
Las piezas cerámicas obtenidas a partir de polvos de ferritas de composición $\left(\mathrm{Cu}_{0.12} \mathrm{Ni}_{0.23} \mathrm{Zn}_{0.65}\right) \mathrm{Fe}_{2} \mathrm{O}_{4}$ destacan como absorbentes de radiación electromagnética entre las ferritas de $\mathrm{Ni}-\mathrm{Zn}$ dopadas con $\mathrm{Cu}$, impidiendo interferencias entre dispositivos electrónicos. En este trabajo se ha establecido una metodología para diseñar la etapa de sinterización de un ciclo térmico, que permite obtener las piezas cerámicas mencionadas con una microestructura adecuada (elevada densidad relativa y escaso crecimiento de grano) para que presenten unas buenas propiedades electromagnéticas.

La metodología establecida consiste, en primer lugar, en determinar las ecuaciones diferenciales que relacionan las velocidades de densificación y de crecimiento de grano con el tamaño medio de grano, la densidad relativa y la temperatura de sinterización, proponiendo en cada caso el mecanismo de difusión controlante de cada una de dichas velocidades. En segundo lugar, la integración simultánea de las dos ecuaciones de velocidad permite obtener la evolución de la densidad relativa y del tamaño medio de grano con el tiempo de sinterización a una temperatura previamente fijada. En tercer lugar, se ha determinado cómo dicha temperatura debe ir disminuyendo con el tiempo de sinterización para que, en cada instante, se mantenga una velocidad de densificación relativamente alta evitando al mismo tiempo que la velocidad de crecimiento de grano provoque un crecimiento irregular y exagerado de los mismos.
\end{abstract}

Palabras clave: ferritas, ciclo térmico, etapa de sinterización, velocidad de densificación, velocidad de crecimiento de grano.

Thermal cycle for obtaining a Ni-Zn ferrite: (I) Design of the sintering stage.

Ceramic bodies formed from $\left(\mathrm{Cu}_{0.12} \mathrm{Ni}_{0.23} \mathrm{Zn}_{0.65}\right) \mathrm{Fe}_{2} \mathrm{O}_{4}$ ferrite powders stand out as electromagnetic radiation absorbers among $\mathrm{Cu}$-doped $\mathrm{Ni}-\mathrm{Zn}$ ferrites, preventing interference between electronic devices. This study establishes a methodology for designing the sintering stage of a thermal cycle, which allows the foregoing ceramic bodies to be obtained with the appropriate microstructure (high relative density and little grain growth) to provide them with good electromagnetic properties.

The methodology consists, first, of determining the differential equations that relate the densification and grain-growth rates to average grain size, relative density, and sintering temperature, proposing the corresponding rate-controlling mass-transfer mechanism in each case. Secondly, the simultaneous integration of the two rate equations allows the evolution of relative density and average grain size with sintering time at a prefixed sintering temperature to be obtained. Thirdly, it is determined how this temperature needs to decrease with sintering time in order constantly to maintain a relatively high densification rate, while concurrently keeping the grain-growth rate from causing irregular and/or exaggerated grain growth.

Keywords: ferrites, thermal cycle, sintering stage, densification rate, grain-growth rate.

\section{INTRODUCCIÓN}

Los polvos cerámicos conocidos genéricamente como ferritas son, básicamente, óxidos mixtos de hierro y de uno o más elementos metálicos como, por ejemplo, manganeso, níquel, cinc, magnesio, cobalto, cobre, tierras raras, bario, estroncio y plomo. Las ferritas de $\mathrm{Ni}-\mathrm{Zn}$, con diferentes porcentajes de $\mathrm{Cu}$, suelen utilizarse para la fabricación de piezas cuya función es la de impedir interferencias entre los dispositivos eléctricos o electrónicos, dada su capacidad de absorción de ondas electromagnéticas (1-4). Las propiedades magnéticas de estas piezas quedan determinadas por su composición química y por su microestructura (distribución de tamaños de grano, distribución de tamaños de poro, porosidad total o densidad relativa, características del borde de grano, etc.) (3-8).

Las piezas de ferrita se fabrican mediante el denominado proceso cerámico tradicional $(6,12)$. Es en la etapa de sinterización donde se debe: i/ conseguir que las piezas alcancen la máxima densidad relativa posible; ii / aumentar el tamaño de los granos de forma homogénea (evitando un crecimiento exagerado), manteniendo un tamaño de grano relativamente pequeño y una distribución de tamaños de grano estrecha (12). La temperatura máxima de sinterización y el tiempo de permanencia a dicha temperatura son las dos variables más relevantes para controlar la sinterización de las ferritas de $\mathrm{Ni}-\mathrm{Zn}(3,7)$.

En una serie de trabajos previos los autores han estudiado la síntesis (9), la sinterización (11, 13-15) y las propiedades electromagnéticas (10) de una ferrita de Ni-Zn de composición $\left(\mathrm{Cu}_{0.25} \mathrm{Ni}_{0.25} \mathrm{Zn}_{0.50}\right) \mathrm{Fe}_{2} \mathrm{O}_{4}$. En este trabajo se ha estudiado la sinterización de piezas, obtenidas por prensado uniaxial en seco de un polvo industrial de ferrita de composición aproximada $\left(\mathrm{Cu}_{0.12} \mathrm{Ni}_{0.23} \mathrm{Zn}_{0.65}\right) \mathrm{Fe}_{2} \mathrm{O}_{4}, \mathrm{y}$, mediante el modelo propuesto por Zhao y Harmer (16-18), se han obtenido tres relaciones matemáticas: una entre la velocidad a la que 
transcurre el proceso de densificación, el tamaño medio de grano y la temperatura; otra entre la velocidad a la que transcurre el proceso de crecimiento de grano, el tamaño medio de grano, la densidad relativa y la temperatura; $y$, finalmente, una tercera entre el tamaño medio de grano, la densidad relativa y la temperatura de sinterización.

Estos resultados han permitido:i/ proponer los mecanismos de transferencia de materia que controlan la velocidad a la que transcurre tanto el proceso de densificación como el de crecimiento de grano; ii / obtener la evolución de la densidad relativa y del tamaño medio de grano con el tiempo de sinterización a una temperatura previamente fijada, mediante la integración simultánea de las dos ecuaciones de velocidad; iii/ diseñar la etapa de sinterización determinando cómo debe ir variando la temperatura con el tiempo de sinterización para que, en cada instante, se mantenga una velocidad de densificación relativamente alta evitando al mismo tiempo que la velocidad de crecimiento de grano provoque un crecimiento irregular y exagerado de los mismos.

\section{PROCEDIMIENTO EXPERIMENTAL}

Como material de partida se ha utilizado un polvo atomizado industrial de ferrita de composición $\left(\mathrm{Cu}_{0.12} \mathrm{Ni}_{0.23}\right.$ $\left.\mathrm{Zn}_{0.65}\right) \mathrm{Fe}_{2} \mathrm{O}_{4}$ con el que se han conformado piezas cilíndricas de $2 \mathrm{~cm}$ de diámetro y $3 \mathrm{~mm}$ de espesor, por prensado en seco unidireccional a una presión de $2000 \mathrm{~kg} / \mathrm{cm}^{2}$ para conseguir una densidad relativa en seco del 0.61 (expresado en tanto por uno respecto a la densidad real del material). Las muestras se sinterizaron en un horno eléctrico de laboratorio, en atmósfera de aire, con un ciclo térmico de tres etapas: una etapa de precalentamiento, a una velocidad de $12^{\circ} \mathrm{C} / \mathrm{min}$, desde temperatura ambiente hasta $500^{\circ} \mathrm{C}$, y un tiempo de permanencia a dicha temperatura de 2 horas para oxidar los aditivos orgánicos utilizados para obtener el polvo atomizado y facilitar la operación de prensado; una etapa de calentamiento, a una velocidad de $12^{\circ} \mathrm{C} / \mathrm{min}$, hasta alcanzar la temperatura máxima de sinterización $(900,950,1000,1025,1050,1075,1100$ y $1200^{\circ} \mathrm{C}$ ) y diferentes tiempos de permanencia (en adelante, tiempo de sinterización) a dicha temperatura; una etapa de enfriamiento súbito (quenching), desde la temperatura máxima de sinterización hasta temperatura ambiente.

El polvo atomizado de ferrita tiene una densidad real $\left(\rho_{\mathrm{r}}\right)$ de $5380 \mathrm{~kg} / \mathrm{m}^{3}$, medida experimentalmente con un picnómetro de helio. De cada pieza sinterizada se ha determinado la densidad aparente $\left(\rho_{\mathrm{ap}}\right)$, mediante el método de Arquímedes por inmersión en mercurio, y la densidad relativa $\left(\phi_{c}\right)$, calculada como cociente entre la densidad aparente $\left(\rho_{\mathrm{ap}}\right)$ y la densidad real del polvo atomizado de ferrita.

El polvo atomizado de ferrita estaba constituido por partículas con un diámetro medio $\left(\mathrm{d}_{50}\right)$ de $0.2 \mu \mathrm{m}$, medido experimentalmente por análisis de imagen de una pieza compactada antes de ser sometida al ciclo de sinterización. En cada pieza sinterizada se ha observado y fotografiado su microestructura: en el caso de piezas con elevada densidad relativa, se pulían con pasta de diamante hasta un acabado de $1 \mu \mathrm{m}$ y posteriormente se sometían a un ataque térmico calentándolas a una temperatura $100-120^{\circ} \mathrm{C}$ inferior a la temperatura de sinterización, durante 30 minutos, con una velocidad de calentamiento de $15^{\circ} \mathrm{C} / \mathrm{min}$, y la superficie atacada térmicamente se observaba y fotografiaba utilizando un microscopio óptico; en el caso de piezas con baja densidad relativa, se fracturaban y la superficie de fractura se observaba y fotografiaba con un microscopio electrónico de barrido. Mediante análisis de imagen de las correspondientes fotografías se ha determinado la distribución de tamaños de grano. De cada fotografía se obtenía una distribución de tamaños de grano discontinua, a partir de la medida del tamaño de múltiples granos, que se transformaba en una distribución continua y se describía utilizando tres diámetros característicos, $G_{10^{\prime}} G_{50}$ y $G_{90}$ los cuales se definen como los tamaños de grano por debajo de los cuales queda un $10 \%$, un $50 \%$ y $90 \%$ en volumen de las partículas totales, respectivamente. Teniendo en cuenta que las distribuciones de tamaños de grano correspondientes a las temperaturas para las cuales ha sido posible aplicar el modelo son distribuciones normales y estrechas, se ha tomado el valor de $G_{50}$ para caracterizar las diferentes distribuciones, con el error que esto puede suponer. A partir de estos datos experimentales se ha calculado para cada pieza la amplitud del intervalo de tamaños de grano, como la diferencia entre $G_{90}$ y $G_{10^{\prime}}$ y el tamaño medio de grano adimensional $(\eta)$, como el cociente entre $G_{50}$ y $_{50}$.

\section{RESULTADOS EXPERIMENTALES}

En la Figura 1 se ha representado la evolución de la densidad relativa $\left(\phi_{c}\right)$ de las piezas frente al tiempo de sinterización, para las ocho temperaturas máximas de sinterización ensayadas. En la Figura 2 se muestra ampliada la zona correspondiente a los tiempos de sinterización comprendidos entre 0 y 5 horas. La curva trazada a cada temperatura únicamente muestra la tendencia de los valores experimentales. No se muestra el error de la medida del valor de la densidad relativa ni del tiempo por ser menores que el tamaño del propio punto. Se observa que al aumentar la temperatura de sinterización aumenta la densidad relativa para un determinado tiempo y que una determinada densidad relativa se alcanza con menor tiempo cuanto mayor es la temperatura. Asimismo, a medida que aumenta la temperatura de sinterización, se produce un incremento de la velocidad de densificación (tangente a la curva en cada punto).

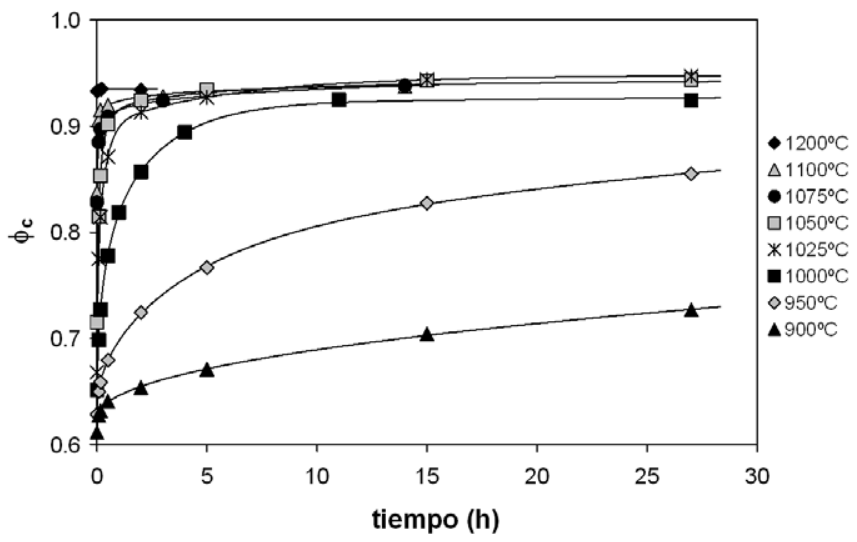

Fig. 1- Evolución de la densidad relativa $\left(\phi_{c}\right)$ con el tiempo de sinterización.

En la Figura 3 se ha representado la evolución del tamaño medio de grano $\left(G_{50}\right)$ de las piezas frente al tiempo de sinterización, para las ocho temperaturas máximas de sinterización ensayadas. La curva trazada a cada temperatura únicamente muestra la tendencia de los valores experimentales. 


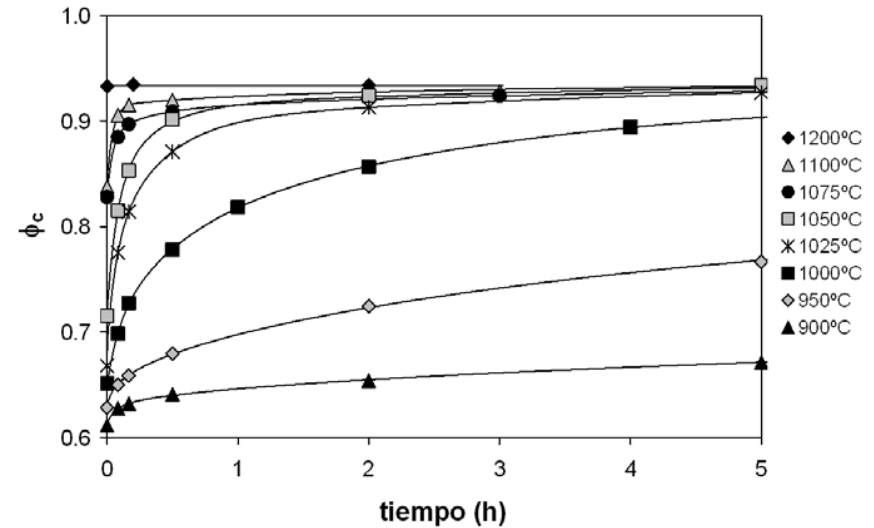

Fig. 2- Evolución de la densidad relativa $\left(\phi_{c}\right)$ con el tiempo de sinterización.

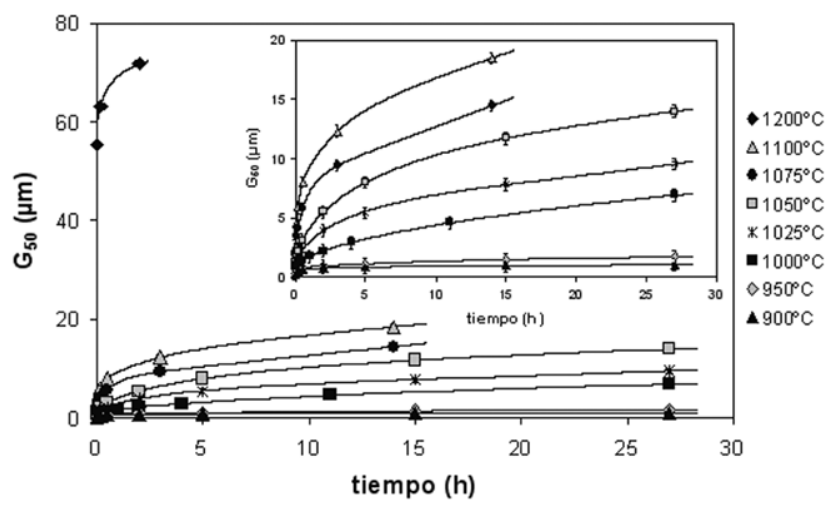

Fig. 3- Evolución del tamaño medio de grano $\left(\mathrm{G}_{50}\right)$ con el tiempo de sinterización.

En cada punto experimental se muestra el error de la medida del valor del tamaño medio de grano, pero no del tiempo por ser, en este último caso, menor que el tamaño del propio punto. Se observa, para temperaturas superiores a $950^{\circ} \mathrm{C}$, que al aumentar la temperatura de sinterización aumenta el tamaño medio de grano para un determinado tiempo y que un determinado tamaño medio de grano se alcanza con menor tiempo cuanto mayor es la temperatura. Asimismo, a medida que aumenta la temperatura de sinterización, se produce un incremento de la velocidad de crecimiento de grano (tangente a la curva en cada punto).

A partir de los datos del tamaño medio de grano mostrados en la Figura 3, en la Figura 4 se ha representado la evolución del tamaño medio de grano adimensional $(\eta)$ de las piezas frente al tiempo de sinterización, para las ocho temperaturas máximas de sinterización ensayadas. La curva trazada a cada temperatura únicamente muestra la tendencia de los valores experimentales.

En la Figura 5 se ha representado el tamaño medio de grano $\left(G_{50}\right)$ frente a la densidad relativa $\left(\phi_{c}\right)$ para las ocho temperaturas ensayadas. Todos los puntos experimentales parecen definir una única curva (18-21). Se observa que hasta alcanzar densidades relativas del orden de 0.9 la sinterización viene acompañada con un ligero crecimiento del tamaño medio de grano. Sin embargo, al alcanzar densidades relativas superiores a 0.9 el crecimiento del tamaño medio de grano se intensifica y la densificación se ralentiza (la densidad relativa tiende asintóticamente hacia un valor aproximado de 0.95$)$. Aunque en la Figura 5 no se muestra la amplitud del intervalo de tamaños de grano correspondiente a cada punto experimental, se ha observado que a medida que se intensifica el crecimiento del tamaño medio de grano (para densidades relativas superiores a 0.9) también aumenta de forma exagerada la amplitud.

Estos resultados ponen de manifiesto la dificultad de sinterizar piezas hasta conseguir una elevada densidad relativa $\left(\phi_{\mathrm{c}} \geq 0.95\right)$ con un escaso crecimiento del tamaño medio de grano y una amplitud del intervalo de tamaños de grano pequeña.

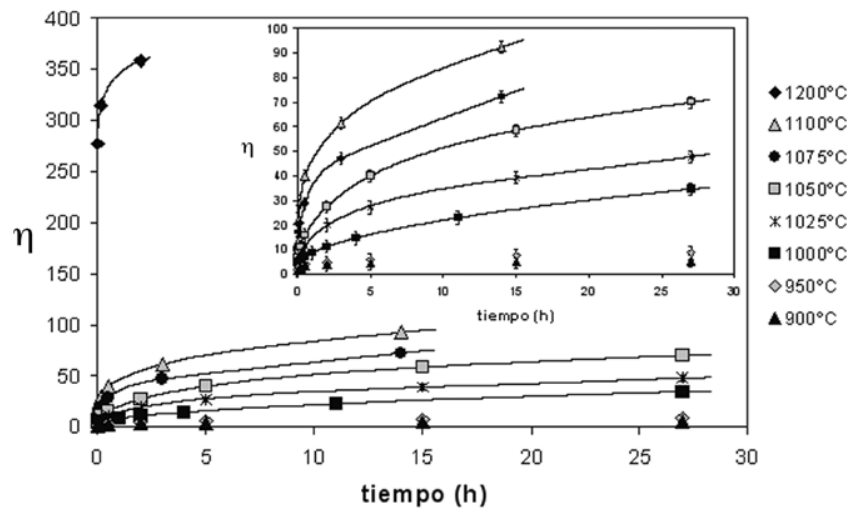

Fig. 4- Evolución del tamaño medio de grano adimensional ( $\eta$ ) con el tiempo de sinterización.

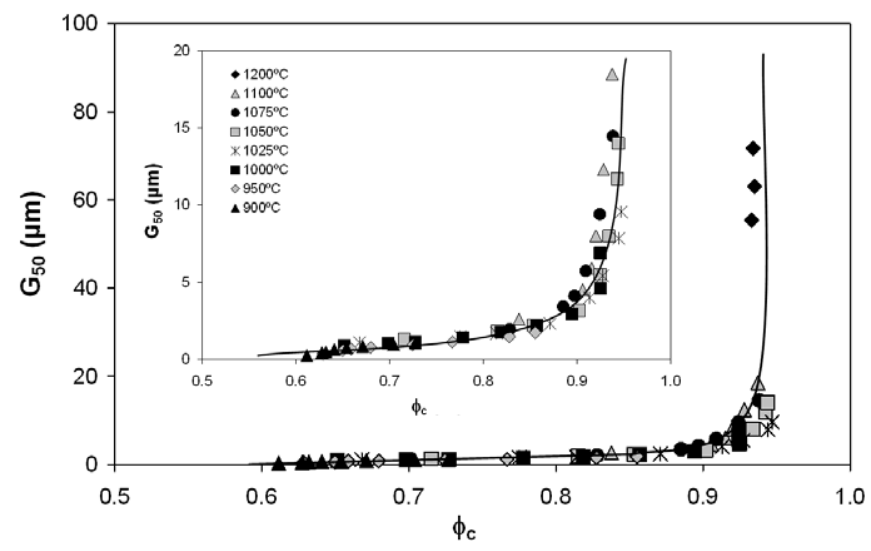

Fig. 5- Evolución del tamaño medio de grano $\left(\mathrm{G}_{50}\right)$ con la densidad relativa $\left(\phi_{\mathrm{c}}\right)$.

\section{ANÁLISIS DE LOS RESULTADOS EXPERIMENTALES}

Durante la sinterización de piezas tienen lugar dos procesos simultáneos y competitivos: el proceso de densificación y el proceso de crecimiento de grano. Para poder obtener una pieza sinterizada con una microestructura adecuada (elevada densidad relativa, pequeño tamaño medio de grano y pequeña amplitud del intervalo de tamaños de grano) que garantice las buenas propiedades del producto final, se debe, en la medida de lo posible, favorecer el proceso de densificación e inhibir el de crecimiento de grano. En consecuencia, resulta muy interesante conocer la velocidad a la que transcurren ambos procesos y las variables de proceso relacionadas. Para 
determinar las velocidades a las que transcurren estos dos procesos se ha utilizado el modelo de sinterización en fase sólida de Zhao y Harmer (16-18), válido para los estadios finales de la sinterización.

\subsection{Velocidad de densificación}

Zhao y Harmer (16-18) han propuesto una ecuación general para la velocidad de densificación de la forma:

$$
\frac{d \phi_{c}}{d t}=\frac{C_{f} \cdot N \cdot D_{f} \cdot \gamma_{s v} \cdot \Omega}{k \cdot T \cdot d_{50}{ }^{h}} \cdot \frac{1}{\eta^{h}}=f \cdot \frac{1}{\eta^{h}}
$$

donde $\mathrm{C}_{\mathrm{f}}$ es una constante relacionada con el mecanismo de control, $\mathrm{N}$ es el número de poros por grano, $\mathrm{D}_{\mathrm{f}}$ es el coeficiente de difusión, $\gamma_{\mathrm{sv}}$ es la energía de la superficie sólido/gas, $\Omega$ es el volumen molar de la especie que se difunde y controla la velocidad de densificación, $\mathrm{k}$ es la constante de Boltzman y $\mathrm{T}$ es la temperatura absoluta. El valor del exponente del tamaño medio de grano adimensional, $h$, es 3 , cuando el mecanismo de transferencia de materia que controla la velocidad de densificación es la difusión reticular, y 4, cuando controla la difusión por el borde de grano. Se puede definir un coeficiente de difusión efectivo $\mathrm{f}$, que debe ser una función compleja de la temperatura ya que engloba constantes $\left(C_{p} \Omega, k, d_{50}{ }^{h}\right)$, parámetros que dependen de la temperatura $\left(\mathrm{N}, \mathrm{D}_{\mathrm{f}}, \gamma_{\mathrm{sv}}\right)$ y la temperatura. Para cada temperatura, $\mathrm{f}$ debe ser una constante. En forma logarítmica la ecuación [1] se puede reescribir como:

$$
\ln \left(\frac{d \phi_{c}}{d t}\right)=\ln (f)-h \cdot \ln (\eta)
$$

La ecuación [2] indica que el logaritmo de la velocidad de densificación, $\ln \left(\mathrm{d} \phi_{\mathrm{c}} / \mathrm{dt}\right)$, debe variar linealmente con el logaritmo del tamaño medio de grano adimensional, $\ln (\eta)$, y que la pendiente de dicha recta es $-h$.

La velocidad de densificación experimental se ha obtenido a partir de las curvas de densidad relativa frente al tiempo de sinterización, mostradas en la Figura 1, correspondientes a las temperaturas de sinterización de 1000, 1025, 1050, 1075 y $1100^{\circ} \mathrm{C}$. Para cada una de las cinco temperaturas indicadas, los puntos experimentales se han ajustado a un polinomio de la forma:

$\phi_{c}=a+\left(n \cdot t^{m}\right)+\left[b \cdot\left(1-\varphi_{1} \cdot e^{-\lambda_{1} \cdot t}-\varphi_{2} \cdot e^{-\lambda_{2} \cdot t}-\varphi_{3} \cdot e^{-\lambda_{3} \cdot t}-\varphi_{4} \cdot e^{-\lambda_{4} \cdot t}\right)\right]$

que posteriormente se ha derivado con respecto al tiempo. De esta forma, para cada punto experimental se ha obtenido su correspondiente velocidad de densificación $\left(\mathrm{d} \phi_{\mathrm{c}} / \mathrm{dt}\right)$.

De acuerdo con la ecuación [2], en la Figura 6 se ha representado el logaritmo de la velocidad de densificación frente al logaritmo del tamaño medio de grano adimensional, quedando definidas cinco rectas. Mediante un ajuste lineal por mínimos cuadrados se han obtenido, para cada recta, los valores de la ordenada en el origen $[\ln (\mathrm{f})]$, la pendiente $(-\mathrm{h}) \mathrm{y}$ el coeficiente de regresión lineal $\left(\mathrm{r}^{2}\right)$ que se detallan en la Tabla I. Como puede observarse en todos los casos el valor de h está próximo a 4, lo que sugiere que, para el material estudiado y en las condiciones ensayadas, el mecanismo de transferencia de materia que controla la velocidad de densificación es la difusión por el borde de grano (16-21).

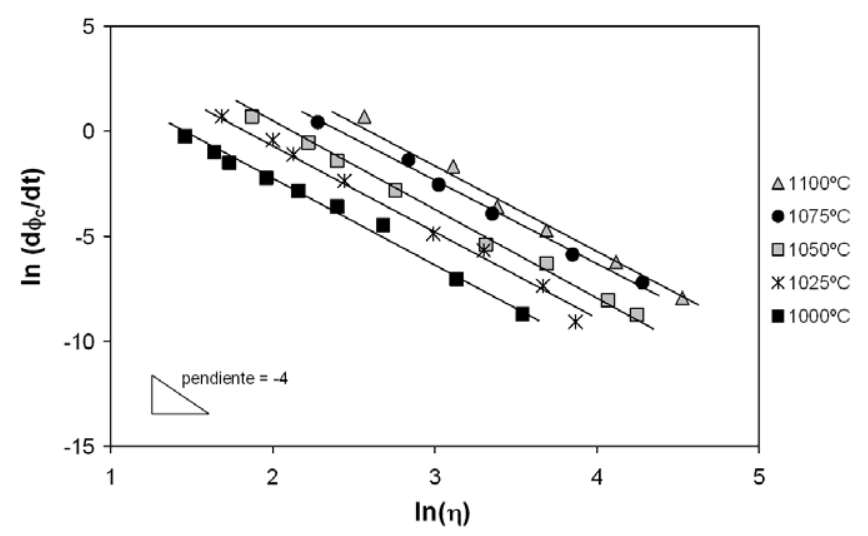

Fig. 6- Relación entre la velocidad de densificación y el tamaño medio de grano adimensional según la ecuación [2].

TABLA I. VALORES DE LOS PARÁMETROS $\ln (\mathrm{f})$ Y h DE LA ECUACIÓN [2].

\begin{tabular}{|c|c|c|c|}
\hline $\mathrm{T}\left({ }^{\circ} \mathrm{C}\right)$ & $\ln (\mathrm{f})$ & $\mathrm{h}$ & $\mathrm{r}^{2}$ \\
\hline 1000 & 6.02 & 4.13 & 0.9883 \\
\hline 1025 & 7.51 & 4.10 & 0.9904 \\
\hline 1050 & 8.95 & 4.22 & 0.9941 \\
\hline 1075 & 9.61 & 3.98 & 0.9943 \\
\hline 1100 & 10.56 & 4.07 & 0.9839 \\
\hline
\end{tabular}

Dado que el coeficiente de difusión efectivo $\mathrm{f}$ es una función complicada y desconocida de la temperatura, se ha propuesto una relación empírica de la forma:

$$
f=A \cdot \exp \left(-\frac{B}{T}\right)
$$

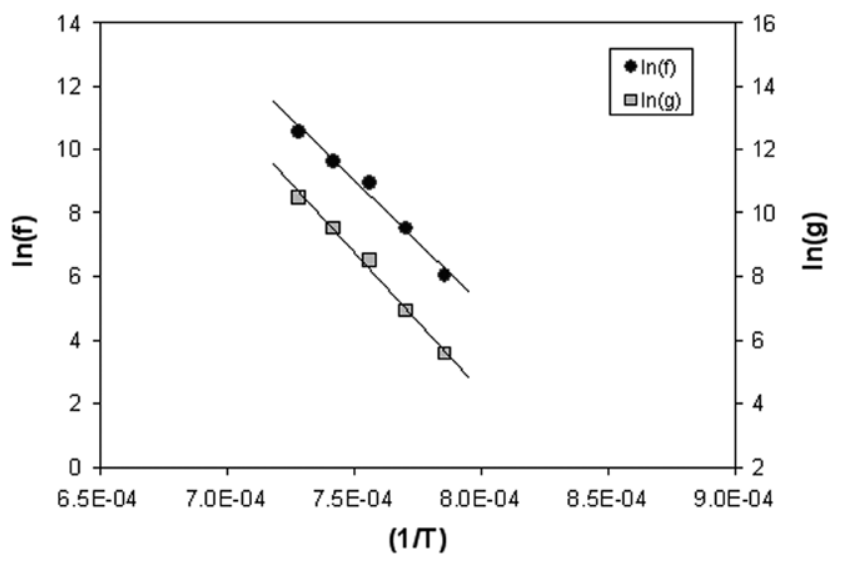

Fig. 7. Relación de los coeficientes de difusión efectivos f y g con la temperatura de sinterización.

En la Figura 7 se han representado los valores del logaritmo de $\mathrm{f}$ frente a la inversa de la temperatura absoluta para las cinco temperaturas mostradas en la Tabla I, obteniéndose una línea recta cuyo ajuste por mínimos cuadrados conduce a la ecuación: 


$$
\ln (f)=68.74-\frac{79604}{T} ; \quad r^{2}=0.9793
$$

o bien:

$$
f=7.11 \cdot 10^{29} \cdot\left[\exp \left(-\frac{79604}{T}\right)\right]
$$

Combinando las ecuaciones (1) y (6) y tomando $\mathrm{h}=4$, la velocidad de densificación de la ferrita estudiada, en las condiciones de operación ensayadas, se puede describir mediante la ecuación:

$$
\frac{\mathrm{d} \phi_{\mathrm{c}}}{\mathrm{dt}}=7.11 \cdot 10^{29} \cdot\left[\exp \left(-\frac{79604}{\mathrm{~T}}\right)\right] \cdot \frac{1}{\eta^{4}}
$$

\subsection{Velocidad de crecimiento de grano}

Zhao y Harmer (16-18) también han propuesto una ecuación general de la velocidad de crecimiento de grano, en el caso de que el crecimiento esté controlado por el arrastre de los poros, de la forma:

$$
\frac{d \eta}{d t}=\left(\frac{C_{g} \cdot N^{q} \cdot D_{g}}{d_{50}{ }^{n+1}}\right) \cdot \frac{1}{\left(1-\phi_{c}\right)^{m}} \cdot \frac{1}{\eta^{n}}=g \cdot \frac{1}{\left(1-\phi_{c}\right)^{m}} \cdot \frac{1}{\eta^{n}}
$$

donde $\mathrm{C}_{\mathrm{g}}$ es un parámetro relacionado con la temperatura de sinterización y con el mecanismo de crecimiento de grano, $\mathrm{D}_{\mathrm{g}}$ es el coeficiente de difusión y $\mathrm{N}$ es el número de poros por grano. Los valores de los exponentes $\mathrm{q}, \mathrm{m}$ y $\mathrm{n}$ dependen del mecanismo de transferencia de materia que controla el arrastre de los poros. Así, $\mathrm{q}=1 / 3, \mathrm{n}=3$ y $\mathrm{m}=4 / 3$ si el mecanismo es la difusión superficial, $\mathrm{q}=0, \mathrm{n}=2$ y $\mathrm{m}=1$ cuando el mecanismo es la difusión reticular o la difusión en fase vapor y $\mathrm{q}=-1 / 3, \mathrm{n}=1$ y $m=2 / 3$ en el caso de que el mecanismo sea la evaporación/ condensación. Se puede definir un coeficiente de difusión efectivo g que debe ser una función compleja de la temperatura ya que engloba constantes $\left(\mathrm{d}_{50}{ }^{\mathrm{n}+1}\right)$ y parámetros que dependen de la temperatura $\left(\mathrm{C}_{\mathrm{g}}, \mathrm{N}, \mathrm{D}_{\mathrm{g}}\right)$. Para cada temperatura, $\mathrm{g}$ debe ser una constante. En forma logarítmica la ecuación [8] se puede reescribir como:

$$
\ln \left(\frac{d \eta}{d t}\right)+m \cdot \ln \left(1-\phi_{c}\right)=\ln (g)-n \cdot \ln (\eta)
$$

La ecuación [9] indica que $\operatorname{In}(\mathrm{d} \eta / \mathrm{dt})+\mathrm{m} \cdot \ln \left(1-\phi_{\mathrm{c}}\right)$ debe variar linealmente con el logaritmo del tamaño medio de grano adimensional, $\ln (\eta)$, y que la pendiente de dicha recta es $-\mathrm{n}$.

La velocidad de crecimiento de grano se ha obtenido a partir de las curvas de tamaño medio de grano adimensional frente al tiempo de sinterización, mostradas en la Figura 4, correspondientes a las temperaturas de sinterización de $1000,1025,1050,1075$ y $1100^{\circ} \mathrm{C}$. Para cada una de las cinco temperaturas indicadas, los puntos experimentales se han ajustado a un polinomio de la forma:

$\eta=a+\left(n \cdot t^{m}\right)+\left[b \cdot\left(1-\varphi_{1} \cdot e^{-\lambda_{1} \cdot t}-\varphi_{2} \cdot e^{-\lambda_{2} \cdot t}-\varphi_{3} \cdot e^{-\lambda_{3} \cdot t}\right)\right]$ que posteriormente se ha derivado con respecto al tiempo. De esta forma, para cada punto experimental se ha obtenido su correspondiente velocidad de crecimiento de grano $(\mathrm{d} \eta / \mathrm{dt})$.

De acuerdo con la ecuación [9] se ha representado $\operatorname{In}(\mathrm{d} \eta /$ $\mathrm{dt})+\mathrm{m} \cdot \ln \left(1-\phi_{\mathrm{c}}\right)$ frente al logaritmo del tamaño medio de grano adimensional, dando sucesivamente a $\mathrm{m}$ los valores de $4 / 3$, 1 y $2 / 3$. Sólo en el caso de $m=4 / 3$ se han obtenido las cinco rectas mostradas en la Figura 8. Mediante un ajuste lineal por mínimos cuadrados se han obtenido, para cada recta, los valores de la ordenada en el origen $[\ln (g)]$, la pendiente $(-n)$ y el coeficiente de regresión lineal $\left(\mathrm{r}^{2}\right)$ que se detallan en la Tabla II. Como puede observarse, en todos los casos el valor de n está próximo a 3, lo que sugiere que, para el material estudiado y en las condiciones ensayadas, el mecanismo de transferencia de materia que controla el arrastre de los poros y, por tanto, la velocidad de crecimiento de grano, es la difusión superficial (16-21).

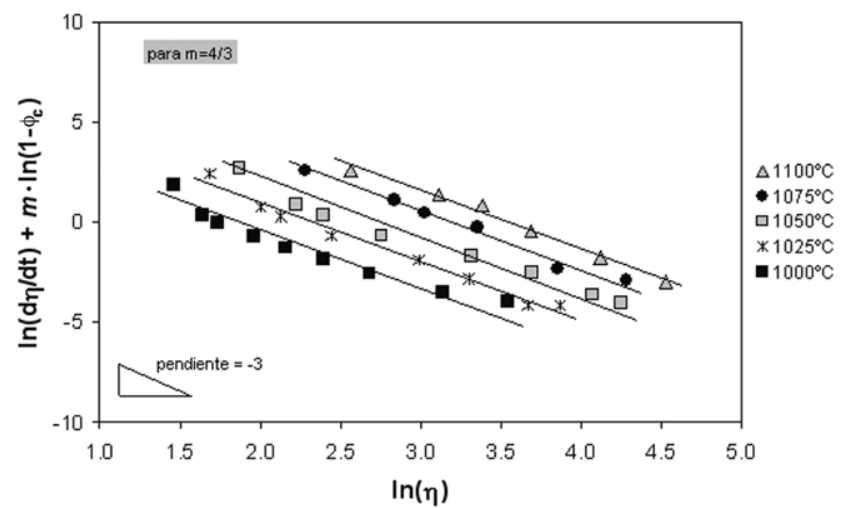

Fig. 8- Relación entre la velocidad de crecimiento de grano y el tamaño medio de grano adimensional según la ecuación [9], con $\mathrm{m}=4 / 3$.

TABLA II. VALORES DE LOS PARÁMETROS In(g) Y n DE LA ECUACIÓN [9]

\begin{tabular}{|c|c|c|c|}
\hline $\mathrm{T}\left({ }^{\circ} \mathrm{C}\right)$ & $\ln (\mathrm{g})$ & $\mathrm{n}$ & $\mathrm{r}^{2}$ \\
\hline 1000 & 5.56 & 2.95 & 0.9216 \\
\hline 1025 & 6.93 & 2.97 & 0.9825 \\
\hline 1050 & 8.51 & 3.08 & 0.9403 \\
\hline 1075 & 9.52 & 2.97 & 0.9857 \\
\hline 1100 & 10.48 & 2.96 & 0.9883 \\
\hline
\end{tabular}

Dado que el coeficiente de difusión efectivo g es una función complicada y desconocida de la temperatura, se ha propuesto una relación empírica de la forma:

$$
g=F \cdot \exp \left(-\frac{J}{T}\right)
$$

En la Figura 7 se han representado los valores del logaritmo de $g$ frente a la inversa de la temperatura absoluta para las cinco temperaturas mostradas en la Tabla II, obteniéndose una línea recta cuyo ajuste por mínimos cuadrados conduce a la ecuación:

$$
\ln (g)=73.38-\frac{86168}{T} ; \quad r^{2}=0.9914
$$


o bien:

$$
g=7.36 \cdot 10^{31} \cdot\left[\exp \left(-\frac{86168}{T}\right)\right]
$$

Combinando las ecuaciones (8) y (13) y tomando $\mathrm{m}=4 / 3$ y $n=3$, la velocidad de crecimiento de grano de la ferrita estudiada, en las condiciones de operación ensayadas, se puede describir mediante la ecuación:

$$
\frac{\mathrm{d} \eta}{\mathrm{dt}}=7.36 \cdot 10^{31} \cdot\left[\exp \left(-\frac{86168}{\mathrm{~T}}\right)\right] \cdot \frac{1}{\left(1-\phi_{c}\right)^{4 / 3}} \cdot \frac{1}{\eta^{3}}
$$

\subsection{Reproducción de los resultados experimentales}

Las ecuaciones (7) y (14), se pueden integrar simultáneamente, mediante el método numérico de RungeKutta-Fehlberg 7-8, con las condiciones iniciales de densidad relativa $\left(\phi_{00}\right)$ y tamaño medio de grano adimensional $\left(\eta_{0}\right)$ (para un tiempo de sinterización igual a cero) mostradas en la Tabla III. Para las temperaturas de sinterización de $1000,1025,1050,1075$ y $1100^{\circ} \mathrm{C}$ se obtienen diez curvas que representan la variación de la densidad relativa con el tiempo de sinterización (Figuras 9 y 10) y la variación del tamaño medio de grano adimensional con el tiempo de sinterización (Figura 11). En las tres figuras se han superpuesto los puntos experimentales observándose una buena concordancia entre dichos valores experimentales y los predichos por las curvas.

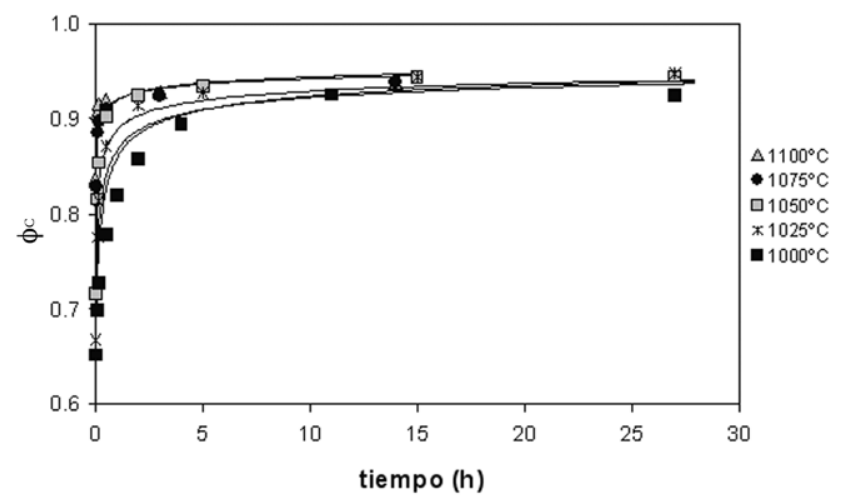

Fig. 9- Concordancia entre los valores experimentales de la densidad relativa y los calculados mediante las ecuaciones [7] y [14].

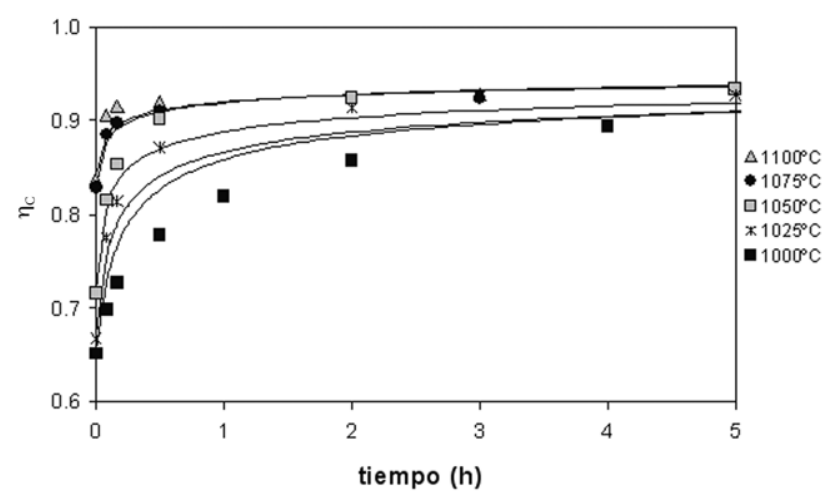

Fig. 10- Concordancia entre los valores experimentales de la densidad relativa y los calculados mediante las ecuaciones [7] y [14].

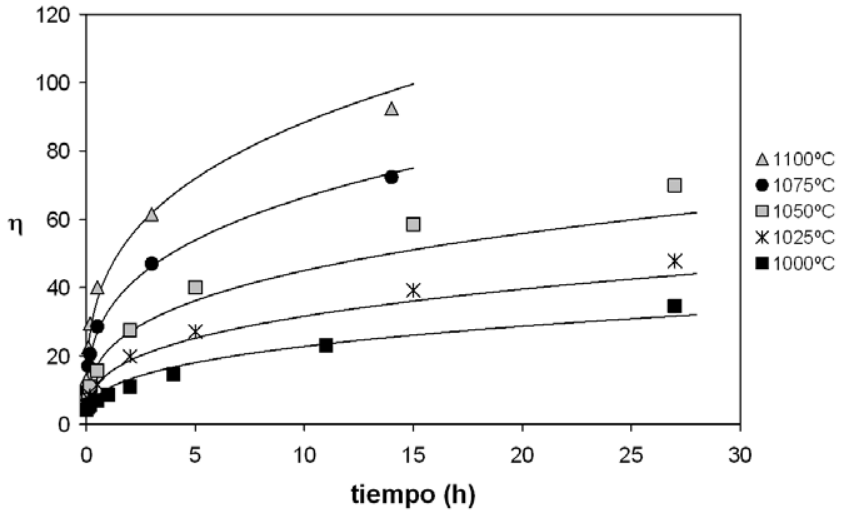

Fig. 11- Concordancia entre los valores experimentales del tamaño medio de grano adimensional y los calculados mediante las ecuaciones [7] y [14].

Al intentar utilizar las ecuaciones (7) y (14) para las temperaturas de sinterización de 900 y $950^{\circ} \mathrm{C}$ no se logra una buena concordancia entre los valores experimentales y los predichos mediante la integración simultánea de las mencionadas ecuaciones. Este hecho puede ser debido a que a las temperaturas de 900 y $950^{\circ} \mathrm{C}$ la sinterización transcurre en la etapa intermedia, sin haberse alcanzado todavía la etapa final de sinterización para la que es válido el modelo de Zhao y Harmer utilizado.

\subsection{Relación entre la densidad relativa y el tamaño medio de grano adimensional}

El cociente entre las ecuaciones (7) y (14) conduce a la expresión:

$\frac{\mathrm{d} \phi_{\mathrm{c}}}{\mathrm{d} \eta}=\frac{7.11 \cdot 10^{29} \cdot\left[\exp \left(-\frac{79604}{\mathrm{~T}}\right)\right]}{7.36 \cdot 10^{31} \cdot\left[\exp \left(-\frac{86168}{\mathrm{~T}}\right)\right]} \cdot \frac{\left(1-\phi_{\mathrm{C}}\right)^{4 / 3}}{\eta}=9.66 \cdot 10^{-3} \cdot \exp \left(\frac{6564}{\mathrm{~T}}\right) \cdot \frac{\left(1-\phi_{\mathrm{c}}\right)^{4 / 3}}{\eta}$

La ecuación [15] permite, para una temperatura de sinterización constante, separar variables e integrar:

$9.66 \cdot 10^{-3} \cdot \exp \left(\frac{6564}{T}\right) \cdot \int_{\phi_{c} 0}^{\phi_{c}}\left(1-\phi_{c}\right)^{4 / 3} \cdot d \phi_{c}=f \int_{\eta_{0}}^{\eta} \eta^{-1} \cdot d \eta$

obteniéndose:

$\eta=\eta_{0} \cdot \exp \left[2.9 \cdot 10^{-2} \cdot \exp \left(\frac{6564}{T}\right) \cdot\left(\frac{1}{\left(1-\phi_{c}\right)^{1 / 3}}-\frac{1}{\left(1-\phi_{c 0}\right)^{1 / 3}}\right)\right]$

Sustituyendo en la ecuación [17] cada una de las cinco temperaturas ensayadas y los valores de la densidad relativa y del tamaño medio de grano adimensional correspondientes al tiempo de sinterización cero $\left(\eta_{0} \mathrm{y} \phi_{\mathrm{c}^{\prime}}\right.$ Tabla III), para cada temperatura, se obtienen las cinco curvas de $\eta=f\left(\phi_{c}\right)$ que se muestran en la Figura 12. En esta figura también se han superpuesto los valores experimentales, observándose una 
buena concordancia entre ellos y los predichos por la ecuación [17]. Las cinco curvas definidas por la ecuación [17], una para cada una de las temperaturas de sinterización ensayadas, se encuentran muy próximas entre sí, definiendo prácticamente una única banda ancha.

TABLA III. VALORES DE $\phi_{\mathrm{c} 0}$ Y $\eta_{0}$ CORRESPONDIENTES AL TIEMPO DE SINTERIZACIÓN INICIAL.

\begin{tabular}{|c|c|c|}
\hline $\mathrm{T}\left({ }^{\circ} \mathrm{C}\right)$ & $\phi_{\mathrm{c} 0}$ & $\eta_{0}$ \\
\hline 1000 & 0.651 & 4 \\
\hline 1025 & 0.668 & 5 \\
\hline 1050 & 0.715 & 7 \\
\hline 1075 & 0.828 & 10 \\
\hline 1100 & 0.838 & 13 \\
\hline
\end{tabular}

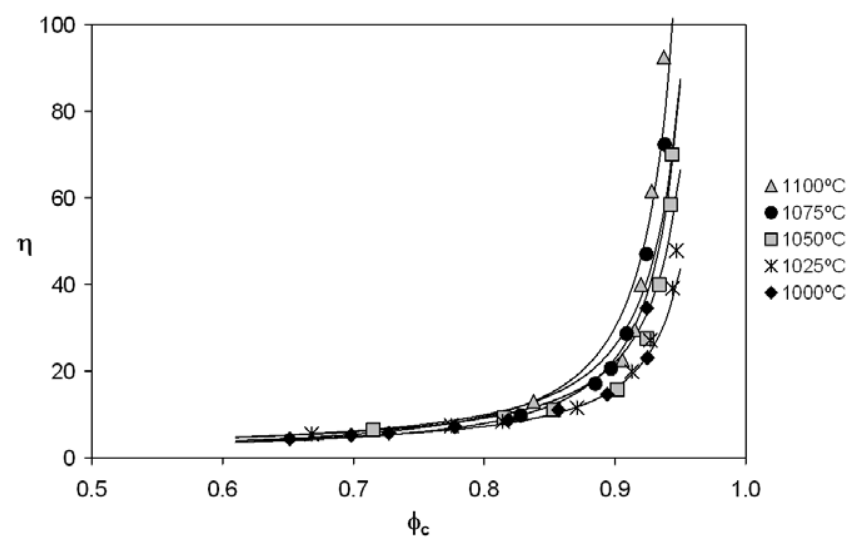

Fig. 12- Concordancia entre los valores experimentales y los calculados mediante las ecuación [17].

\subsection{Relación entre los coeficientes de difusión efectivos}

El factor $\Gamma$, definido como cociente entre los coeficientes de difusión efectivos del proceso de densificación (ecuación [6]) y del proceso de crecimiento de grano (ecuación [13]), depende de la temperatura de sinterización según la expresión:

$$
\Gamma=\frac{f}{g}=\frac{7.11 \cdot 10^{29} \cdot \exp \left[-\frac{79604}{T}\right]}{7.36 \cdot 10^{31} \cdot \exp \left[-\frac{86168}{T}\right]}=9.67 \cdot 10^{-3} \cdot \exp \left[\frac{6564}{T}\right]
$$

Es decir, en el caso de la ferrita estudiada, para las condiciones de operación ensayadas y en el intervalo de temperaturas estudiado (entre 1000 y $1100^{\circ} \mathrm{C}$ ), $\Gamma$ permanece prácticamente constante e independiente de la temperatura de sinterización. Esta escasa influencia de la temperatura de sinterización sobre el factor $\Gamma$ ya se había puesto de manifiesto en la Figura 7, donde se observa que los dos coeficientes de difusión efectivos evolucionan de forma prácticamente paralela con la inversa de la temperatura absoluta y además sus valores están muy próximos en todo el intervalo de temperaturas. Asimismo, justifica que los valores experimentales de $\eta$ frente a $\phi_{c}$ definan una banda ancha, prácticamente independiente de la temperatura de sinterización, como se apreciaba en las Figuras 5 y 12.

\section{DISEÑO DE LA ETAPA DE SINTERIZACIÓN}

Las ecuaciones (7) y (14) indican que para obtener piezas de ferrita de la máxima densidad posible se debería realizar la sinterización a la temperatura máxima permisible, para que la velocidad de densificación (ecuación [7]) fuera elevada. Sin embargo, si se mantuviera dicha temperatura elevada durante un tiempo prolongado resultaría inevitable que también la velocidad de crecimiento de grano (ecuación [14]) fuera muy elevada, provocando un crecimiento exagerado de los granos. Por lo tanto, una etapa de sinterización a una temperatura elevada y constante no resulta adecuada puesto que conduciría a piezas de elevada densidad pero con microestructura muy heterogénea que, a su vez, impediría que las piezas presentaran las propiedades magnéticas deseadas (8, 22-23). Las ecuaciones [15] y [18] también ponen de manifiesto que, en el intervalo de condiciones estudiado, la velocidad de crecimiento de grano siempre es mayor que la velocidad de densificación, lo que supone un problema adicional a la hora de diseñar la etapa de sinterización.

A la vista de las dos consideraciones anteriores se ha diseñado la etapa de sinterización siguiendo dos principios: i/ densificar a temperatura elevada y constante durante un cierto tiempo, determinado por la restricción de que el tamaño medio de grano no crezca excesivamente (tiempo máximo de permanencia a una temperatura, $\mathrm{t}_{\max , \mathrm{T}}$ ); ii / alcanzado este tiempo, disminuir la temperatura de sinterización y mantenerla nuevamente constante durante otro cierto tiempo, determinado por la misma restricción (al disminuir la temperatura se reducen tanto la velocidad de densificación como la de crecimiento de grano pero se consigue mantener el proceso de densificación, a velocidades lo más alta posibles, controlando el tamaño de grano para que no se produzca un crecimiento exagerado del mismo). Este proceso de disminución de la temperatura de sinterización se puede realizar cuantas veces sea necesario siempre que se mantengan valores aceptables de la velocidad de densificación.

Para calcular el tiempo máximo de permanencia a cada temperatura, primero se integran simultánea y numéricamente (mediante el método Runge-Kutta-Fehlberg 7-8) las ecuaciones [7] y [14], a la mencionada temperatura, con las condiciones iniciales correspondientes (los valores de $\phi_{\mathrm{c} 0}$ y $\eta_{0}$ que correspondan al instante en que se alcanza la temperatura, ver Tabla III); se obtienen así ternas de valores de $t, \phi_{c}$ y $\eta$ que representan la evolución de la densidad relativa y del tamaño medio de grano adimensional con el tiempo. A continuación cada terna de valores $t, \phi_{\mathrm{c}}$ y $\eta$ se sustituye en las ecuaciones [7] y [14] obteniéndose otro conjunto de ternas de valores de $\mathrm{t}, \mathrm{d} \phi_{\mathrm{c}} / \mathrm{dt}$ y $\mathrm{d} \eta / \mathrm{dt}$ que representan la evolución de las velocidades de densificación y de crecimiento de grano con el tiempo y que son curvas análogas a las que cualitativamente se muestran en la Figura 13. Finalmente, a partir de cada una de las dos curvas representadas en la Figura 13 se puede calcular el tiempo al que se presenta el vértice de cada una de las curvas de velocidad como el tiempo que corresponde al punto de corte de las rectas tangentes a las ramas de las curvas de velocidad. Como puede observarse en la Figura 13, se obtiene un tiempo para la curva de velocidad de densificación $\left(\mathrm{t}_{\mathrm{d}}\right)$ y otro para la curva de velocidad de crecimiento de grano $\left(\mathrm{t}_{\mathrm{g}}\right)$. El menor de ambos se identifica como el tiempo máximo de permanencia a la temperatura indicada $\left(t_{\max , T}\right)$. La elección de este valor como $t_{\max , \mathrm{T}}$ se deba a : i/ representa el valor al que las velocidades de crecimiento de grano y de densificación 
dejan de disminuir rápidamente con el tiempo y pasan a variar muy lentamente (Figura 13). Siendo este efecto mas considerable cuanto mayor es la temperatura de sinterización, y ii / como puede observarse en las Figuras 14 a 18, a partir de dicho valor las diferencias entre la velocidad de densificación y la de crecimiento de grano $\left(\mathrm{d} \eta / \mathrm{dt}-\mathrm{d} \phi_{\mathrm{c}} / \mathrm{dt}\right)$ se hacen cada vez más grandes.

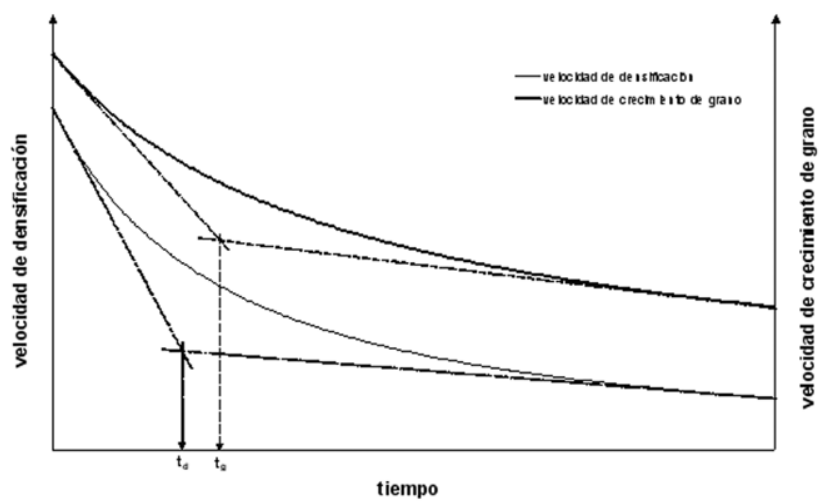

Fig. 13-Cálculo del tiempo máximo de permanencia a una temperatura de sinterización.

En este trabajo se ha aplicado el procedimiento descrito en el párrafo anterior a seis temperaturas (1100, 1080, 1060, 1040, 1020 y $1000^{\circ} \mathrm{C}$ ), comenzando a sinterizar por la temperatura de $1100^{\circ} \mathrm{C}$ y disminuyendo la temperatura de sinterización una vez que se alcanzaba el tiempo máximo de permanencia $\left(t_{\max , T}\right)$. En la Tabla IV se muestran, para cada temperatura, los valores iniciales de $\phi_{\mathrm{c} 0}$ y $\eta_{0}$ necesarios para proceder a la integración simultánea y numérica de las ecuaciones [7] y [14]. Obsérvese que en el caso de $1100^{\circ} \mathrm{C}$, estas condiciones iniciales coinciden con las mostradas en la Tabla III, por ser la temperatura a la que se inicia la simulación, mientras que en el resto de las temperaturas, dichas condiciones iniciales coinciden con los valores que esos parámetros tenían al alcanzar el tiempo máximo de permanencia a la temperatura inmediatamente superior. Asimismo, se muestran los valores de $t_{d}$ y $t_{g}$ (calculados a partir de las Figuras 14 a 19), el tiempo máximo de permanencia a cada temperatura $\left(\mathrm{t}_{\text {max, } \mathrm{T}}\right)$, igual al menor entre $t_{d}$ y $t_{g^{\prime}}$ y el tiempo de sinterización (tiempo acumulado o sumatorio de los tiempos máximos de

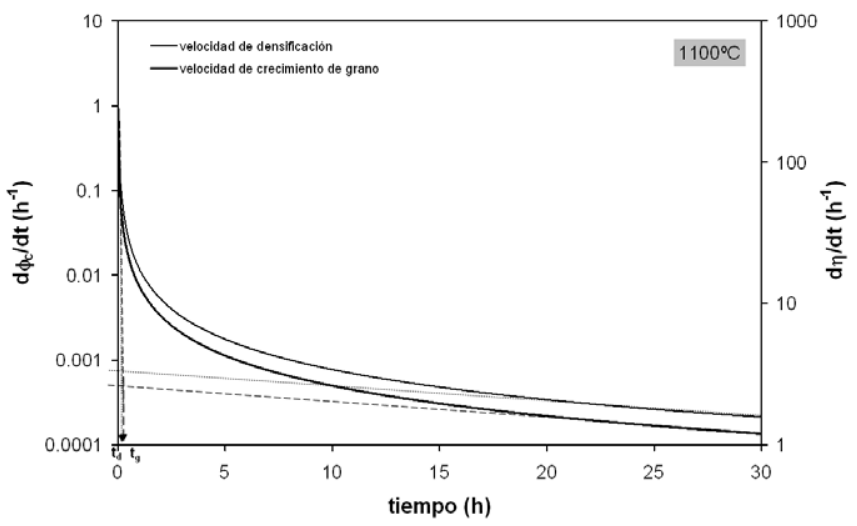

Fig. 14-Cálculo del tiempo máximo de permanencia a $1100^{\circ} \mathrm{C}$.

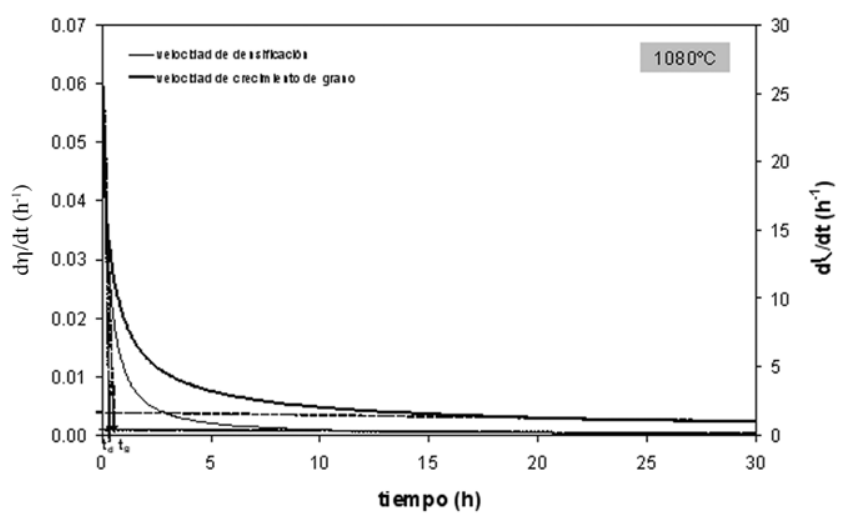

Fig. 15-Cálculo del tiempo máximo de permanencia a $1080^{\circ} \mathrm{C}$.

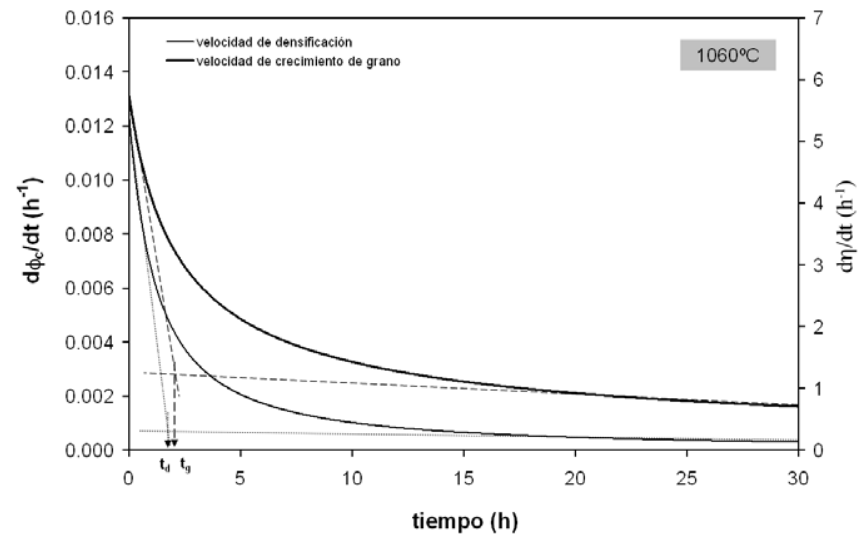

Fig. 16-Cálculo del tiempo máximo de permanencia a $1060^{\circ} \mathrm{C}$.

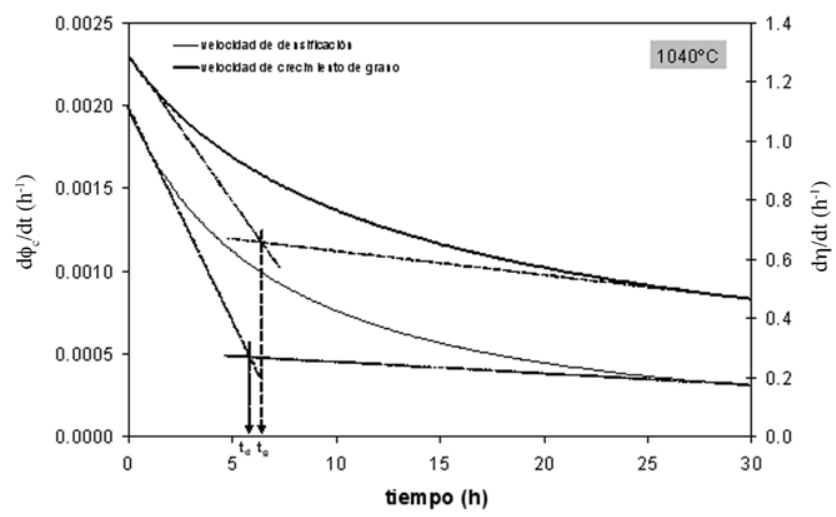

Fig. 17-Cálculo del tiempo máximo de permanencia a $1040^{\circ} \mathrm{C}$.

permanencia a cada temperatura). Finalmente, en esta tabla también se incluye el tamaño medio de grano $\left(G_{50}=\eta \cdot d_{50}\right)$ que presenta una pieza para cada uno de los tiempos de sinterización $(\mathrm{t})$, poniéndose de manifiesto que no llega a superar los $10 \mu \mathrm{m}$.

El análisis de los resultados mostrados en la Tabla IV conduce a las siguientes conclusiones: i/ disminuyendo gradualmente la temperatura de sinterización y aumentando el tiempo de permanencia a cada temperatura, se consigue aumentar la densidad relativa de piezas de la ferrita estudiada hasta una valor del 0.93 con un crecimiento del tamaño medio de grano muy controlado y que no sobrepasa el valor de 


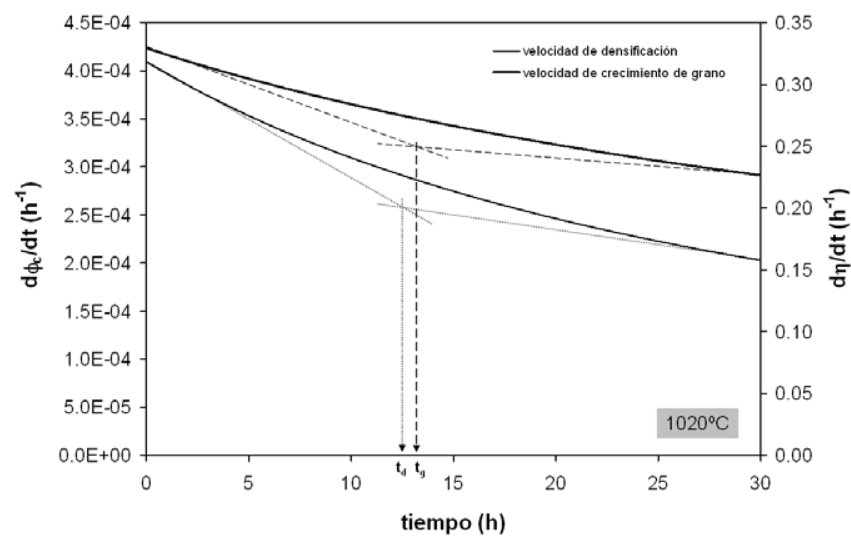

Fig. 18-Cálculo del tiempo máximo de permanencia a $1020^{\circ} \mathrm{C}$

$10 \mu \mathrm{m}$; ii/ la densidad relativa alcanza el valor máximo a la temperatura de $1020^{\circ} \mathrm{C}$, no siendo interesante disminuir dicha temperatura puesto que ya no se consigue incrementar la densificación (probablemente porque la velocidad de densificación ya es excesivamente pequeña) mientras que sigue aumentando el tamaño medio de grano y el tiempo de la etapa de sinterización se alarga desde 20.4 hasta 35.9 horas (véase los valores correspondientes a la temperatura de $\left.1000^{\circ} \mathrm{C}\right)$.

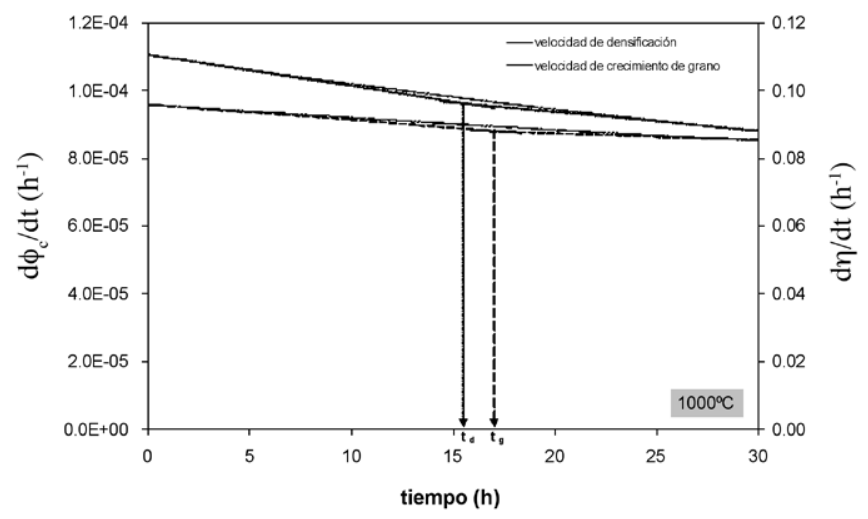

Fig. 19-Cálculo del tiempo máximo de permanencia a $1000^{\circ} \mathrm{C}$.

Sin embargo, teniendo en cuenta que los ciclos térmicos industrialmente utilizados para sinterizar este tipo de materiales se desarrollan a temperaturas máximas entorno a los $1100^{\circ} \mathrm{C}$, durante unas 9-10 horas, y viendo que el último tramo a temperatura constante simulado apenas modifica la microestructura del material y por el contrario alarga el ciclo térmico más de 12 horas, se debería despreciar también el tramo a la temperatura de $1020^{\circ} \mathrm{C}$.

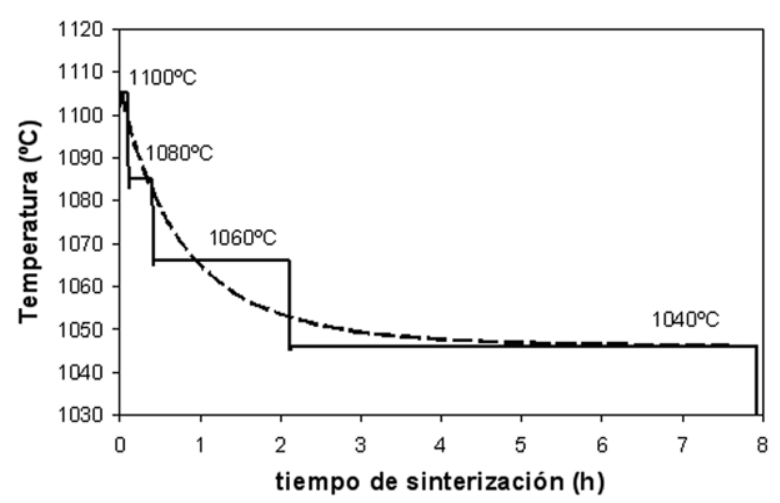

Fig. 20-Etapa de sinterización.

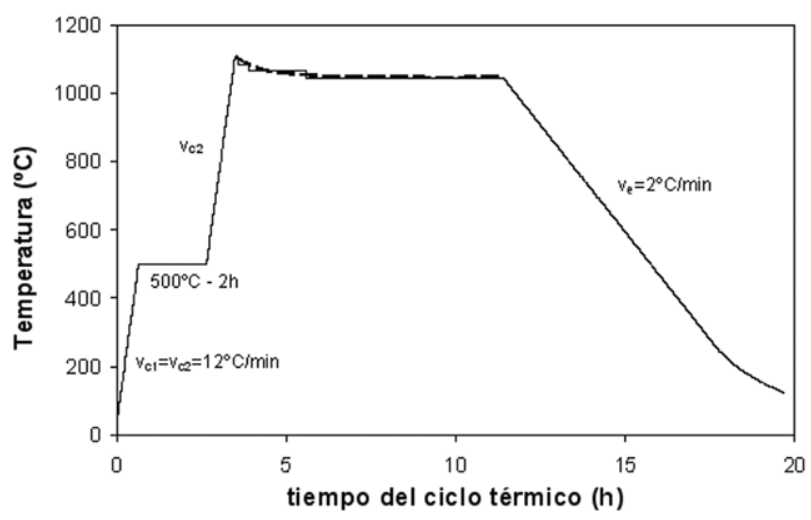

Fig. 21-Ciclo térmico.

En la Figura 20 se ha representado la temperatura de sinterización frente al tiempo de sinterización (columnas primera y novena de la Tabla IV), considerando sólo los cuatro primeros puntos, lo que constituye la etapa de sinterización del ciclo térmico diseñado. En la Figura 21 se muestra la mencionada etapa de sinterización integrada en el ciclo térmico completo utilizado (etapas de calentamiento, hasta alcanzar la etapa de sinterización, y de enfriamiento, después de la etapa de sinterización) en este trabajo. Los cuatro tramos, correspondientes a las cuatro temperaturas, propuestos para la etapa de sinterización, se pueden sustituir por una función continua que disminuya gradualmente la temperatura de sinterización a medida que avanza el tiempo de sinterización. Esta función se ha determinado empíricamente, se puede representar mediante la expresión:

$$
T=\left(20712 \cdot \mathrm{t}^{-4.6}\right)+\left[1046 \cdot\left(1-\mathrm{e}^{-15.4 \cdot \mathrm{t}}\right)\right]
$$

y se ha representado también en las Figuras 20 y 21 en forma de curva discontinua.

TABLA IV. VALORES DE LOS PARÁMETROS UTILIZADOS EN EL DISEÑO DE LA ETAPA DE SINTERIZACIÓN

\begin{tabular}{|c|c|c|c|c|c|c|c|c|c|}
\hline $\mathrm{T}\left({ }^{\circ} \mathrm{C}\right)$ & $\phi_{c 0}$ & $\eta_{0}$ & $\mathrm{t}_{\mathrm{d}}(\mathrm{h})$ & $\mathrm{t}_{\mathrm{g}}(\mathrm{h})$ & $\mathrm{t}_{\text {max } \mathrm{T}}(\mathrm{h})$ & $\phi_{c}$ & $\eta$ & $\mathrm{t}_{\mathrm{c}}(\mathrm{h})$ & $\mathrm{G}_{50}(\mu \mathrm{m})$ \\
\hline 1100 & 0.838 & 13 & 0.1 & 0.2 & 0.1 & 0.89 & 23.1 & 0.1 & 4.6 \\
\hline 1080 & 0.89 & 23.1 & 0.3 & 0.5 & 0.3 & 0.90 & 28.6 & 0.4 & 5.7 \\
\hline 1060 & 0.90 & 28.6 & 1.7 & 2.0 & 1.7 & 0.91 & 36.0 & 2.1 & 7.2 \\
\hline 1040 & 0.91 & 36.0 & 5.8 & 6.4 & 5.8 & 0.92 & 42.2 & 7.9 & 8.4 \\
\hline 1020 & 0.92 & 42.2 & 12.5 & 13.2 & 12.5 & 0.93 & 46.0 & 20.4 & 9.2 \\
\hline 1000 & 0.93 & 46.0 & 15.5 & 17.1 & 15.5 & 0.93 & 47.4 & 35.9 & 9.5 \\
\hline
\end{tabular}


En la Figura 22 se ha representado la evolución de las velocidades de crecimiento de grano y de densificación en función del tiempo de sinterización para la temperatura inicial de $1100^{\circ} \mathrm{C}$. Asimismo, se ha incluido en esta gráfica la evolución de ambas velocidades durante el ciclo térmico diseñado (CTD). Como puede observarse, la disminución progresiva de la temperatura máxima de sinterización supone una disminución considerable de la velocidad de crecimiento de grano pero sin sacrificar en gran medida la velocidad de densificación, lo cual debería permitir densificar la pieza hasta valores próximos a la densidad real del material, controlando el tamaño de los granos.

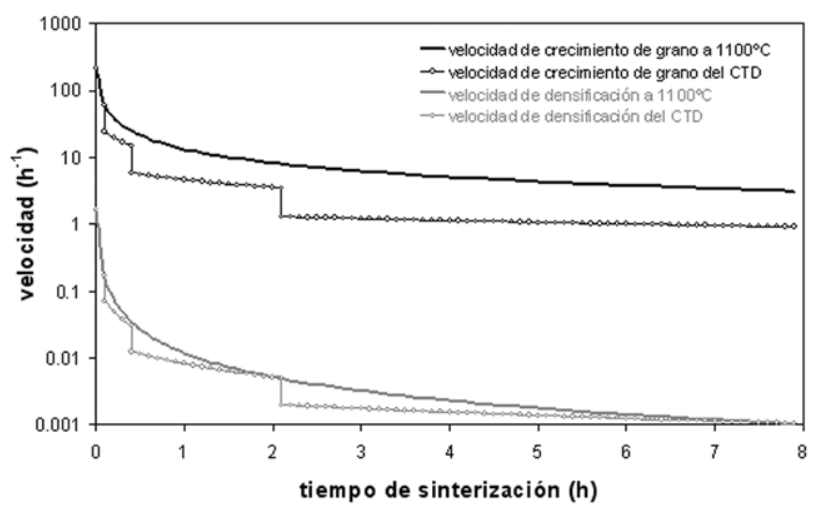

Fig. 22-Evolución de la velocidad de crecimiento de grano y de la velocidad de densificación con el tiempo de sinterización a la temperatura máxima de $1100^{\circ} \mathrm{C}$ y para el ciclo térmico diseñado (CTD).

Efectivamente, al sinterizar una pieza de ferrita, compactada a una densidad relativa en seco del 0.61 , con el ciclo térmico propuesto mostrado en las Figuras 20 y 21, se obtiene una pieza de densidad relativa en cocido de 0.955 y de tamaño medio de grano de $11.8 \mu \mathrm{m}$, cuya microestructura en cocido se muestra en la Figura 23.

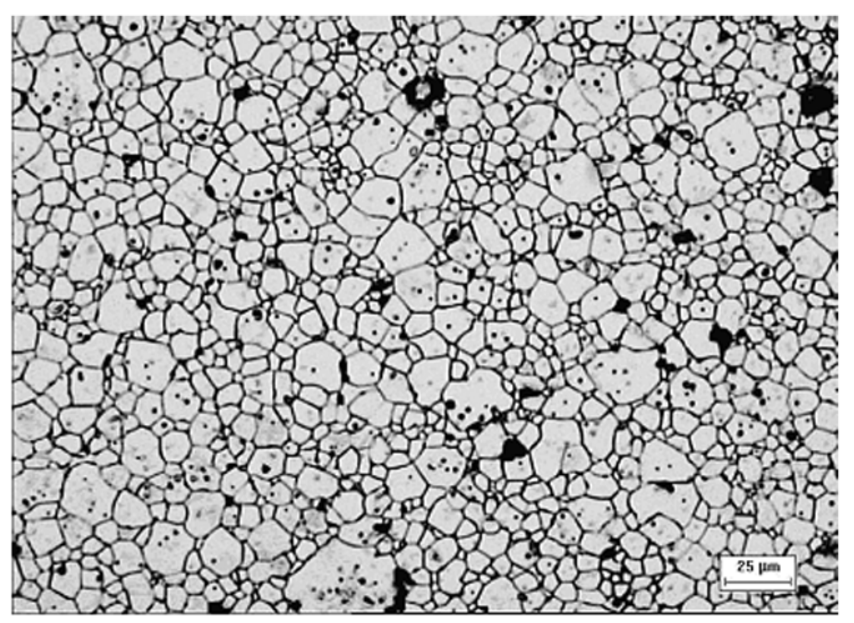

Fig. 23-Microestructura de la pieza obtenida con el ciclo térmico diseñado (CTD).

En la Figura 24 se muestra la microestructura de una pieza comercializada de una ferrita similar a la utilizada para el desarrollo del estudio llevado a cabo. Como puede apreciarse, la microestructura de la pieza industrial resulta análoga a la obtenida con el ciclo térmico diseñado, siendo su densidad relativa (0.942) y su tamaño medio de grano $(11.0 \mu \mathrm{m})$ también similar.

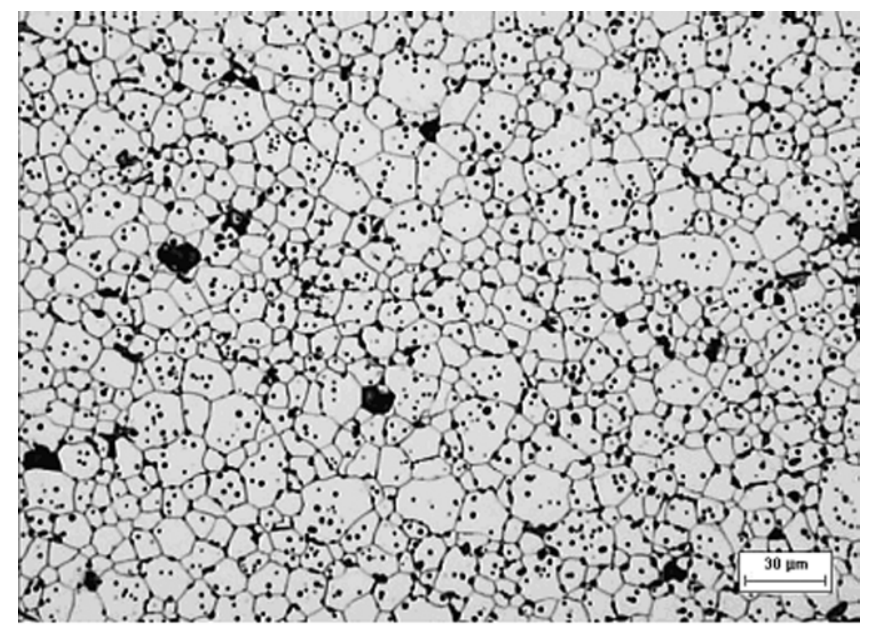

Fig. 24-Microestructura de la pieza industrial.

\section{CONCLUSIONES}

Para la ferrita de composición $\left(\mathrm{Cu}_{0.12} \mathrm{Ni}_{0.23} \mathrm{Zn}_{0.65}\right) \mathrm{Fe}_{2} \mathrm{O}_{4}$ y en las condiciones de operación estudiadas:

1. Se ha relacionado (ecuaciones [7] y [14]) la velocidad de densificación y la velocidad de crecimiento de grano con la temperatura de sinterización, el tamaño medio de grano adimensional y la densidad relativa.

2. Se ha encontrado que el proceso de densificación parece estar controlado por el transporte de materia vía difusión por el borde de grano, mientras que el proceso de crecimiento de grano parece estar controlado por el transporte de materia vía difusión superficial.

3. Se ha obtenido la relación de cada uno de los coeficientes de difusión efectivos con la temperatura (ecuaciones [6] y [13]) y se ha encontrado que el factor $\Gamma$ (su cociente) permanece prácticamente constante con la temperatura.

4. Se ha obtenido una relación matemática (ecuación [17]) entre el tamaño medio de grano adimensional $(\eta)$, la densidad relativa en cocido $\left(\phi_{c}\right)$ y la temperatura de sinterización.

5. Se ha establecido una metodología para diseñar, a partir de las ecuaciones de velocidad de la densidad relativa en cocido $\left(\phi_{c}\right)$ y del tamaño medio de grano adimensional $(\eta)$, la etapa de sinterización del ciclo térmico, es decir, la evolución de la temperatura de sinterización con el tiempo.

6. Se ha diseñado una etapa de sinterización, basada en una disminución de la temperatura a medida que avanza el tiempo, que ha permitido obtener piezas de ferrita de elevada densidad relativa y con un crecimiento de los granos controlado y homogéneo.

\section{BIBLIOGRAFÍA}

1. K.Y. Kim, W.S. Kim, Y.D. Ju, H.J. Jung. Effect of addition of the $\mathrm{CuO}-\mathrm{Fe}_{2} \mathrm{O}_{3}$ system on the electromagnetic wave absorbing properties of sintered ferrite, J. Mater. Sci. 27, 4741-4745 (1992) 
2. A.M. El-Sayed. Effect of chromium substitutions on some properties of NiZn ferrites. Ceram. Int. 28 6, 651-655 (2002)

3. W.S. Kim, S.J. Yoon, K.Y. Kim. Effects of sintering conditions of sintered Ni$\mathrm{Zn}$ ferrites on properties of electromagnetic wave absorber. Mater Lett. 19, 3-4, 149-155 (1994)

4. S.R. Murthy. Low temperature sintering of NiCuZn ferrite and its electrical, magnetic and elastic properties. J. Mater. Sci. Lett. 21, 8, 657-660 (2002)

5. H. Igarashi, K. Okazaki. Effects of porosity and grain size on the magnetic properties of NiZn ferrite. J. Am. Ceram. Soc. 60, 1-2, 51-54 (1977)

6. J. Sanyal. Manufacture of ferrites, pp. 496-505 in S. KUMAR. (ed. lit.) Handbook of ceramics: section B. Kumar \& associates, Calcutta, 1995.

7.SH. Bashkirov, A.B. Liberman, A.M. Khasanov, Effect of sintering temperature on crystalline and magnetic structure of nickel zinc derrites, Soviet Powder Metallurgy and Metal Ceramics (EnglishTranslation), 27, 5, 367-370 (1988).

8. F.Y. Man, Microstructural control in the processing of electronic ceramics, Mater. Sci. Eng. 48, 53-72 (1981).

9. A. Barba, M.J. Orts, E. Sánchez, C. Clausell, Kinetic model applicable to synthesis of $\left(\mathrm{Cu}_{025} \mathrm{Ni}_{025} \mathrm{Zn}_{050}\right) \mathrm{Fe}_{2} \mathrm{O}_{4}$ ferrite, Br. Ceram. Trans. 99, 2, 53-56 (2000).

10. A. Barba, C. Clausell, C. Felíu, M. Monzó, L. Nuño, D. Heras, J.V. Balbastre Study of NiZn Ferrite Complex Permeability: Effect of relative density and microstructure, J. Am. Ceram. Soc. 87, 7, 1314-1318 (2004)

11. A. Barba, C. Clausell, C. Felíu, M. Monzó, Influencia del tamaño de partícula, de la microestructura en crudo y del tratamiento térmico sobre la microestructura en cocido de una ferrita de Ni-Zn, pp. 317-324 en VIII Congreso Nacional de Materiales: Universidad Politécnica de Valencia, Valencia, 2004

12. J. Kulikowski, A. Lesniewski, Properties of Ni-Zn ferrites for magnetic heads: technical possibilities and limitations, J. Magn. Magn. Mater. 19, 117-119 (1980).

13. A. Barba, C. Clausell, C. Felíu, M. Monzó, Sintering of $\left(\mathrm{Cu}_{0.25} \mathrm{Ni}_{0.25} \mathrm{Zn}_{0.50}\right)$ $\mathrm{Fe}_{2} \mathrm{O}_{4}$ Ferrite, J. Am. Ceram. Soc. 87, 4, 571-577 (2004)
14. A. Barba, C. Clausell, M. Monzó, V. Cantavella, Study of the sintering of ferrites of composition $\left(\mathrm{Cu}_{0.25} \mathrm{Ni}_{0.25} \mathrm{Zn}_{0.50}\right) \mathrm{Fe}_{2} \mathrm{O}_{4^{\prime}}$ pp. 185 en $\mathrm{X}$ Congreso Mediterráneo de Ingeniería Química: Ingeniería química y vida: abstracts: Fira de Barcelona, Barcelona, 2005.

15. A. Barba, C. Clausell, M. Monzó, V. Cantavella, Diseño del ciclo térmico de una ferrita, de composición $\left(\mathrm{Cu}_{0.25} \mathrm{Ni}_{0.25} \mathrm{Zn}_{0.50}\right) \mathrm{Fe}_{2} \mathrm{O}_{4^{\prime}}$ basado en su cinética de sinterización, en A. Ramírez de Arellano et. al. (Eds.) Innovación, ciencia y tecnología de los materiales cerámicos y vítreos 2005: Libro de resúmenes de los trabajos presentados a la XLV reunión anual de la Sociedad Española de Cerámica y Vidrio: SECV, Sevilla, 2005.

16. J. Zhao, M.P. Harmer. Effect of pore distribution on microstructure development: I matrix pores, J. Am. Ceram. Soc. 71, 2, 113-120 (1988)

17. J. Zhao, M.P. Harmer, Effect of pore distribution on microstructure development: II, first and second-generation pores, J. Am. Ceram. Soc. 71, 7, 530-539 (1988).

18. J. Zhao, M.P. Harmer, Effect of pore distribution on microstructure development: III model experiments. J. Am. Ceram. Soc. 75, 4, 830-843 (1992).

19. S.A. Nightingale, H.K. Worner, D.P. Dunne, Microstructural development during the microwave sintering of yttria-zirconia ceramics, J. Am. Ceram. Soc. 80, 2, 394-400 (1997).

20. T. Jardiel, A.C. Caballero, M. Villegas, Sintering kinetic of $\mathrm{Bi}_{4} \mathrm{Ti}_{3} \mathrm{O}_{12}$ based ceramics, Bol. Soc. Esp. Ceram. V., 45, 3, 202-206, 2006

21. C. García Gañán, E. Zapata Solvas, D. Gómez García, A. Domínguez Rodríguez, Crecimiento de grano y comportamiento mecánico a alta temperatura de circona itriada (YTZP 4 MOL \%), Bol. Soc. Esp. Ceram. V., 44, 5, 301-306, 2005

22. R.J. Brook, Fabrication principles for the production of ceramics with superior mechanical properties, pp. 7-24 in R.W. Davidge (Ed.) Engineering with ceramics. Stoke-on-Trent: Br. Ceram. Soc. 1982. (Proceedings of the British Ceramic Society ; 32)

23. A.M. Glaeser, Investigating surface transport in ceramics using microdesigned interfaces, pp. 241-282 in R. Smart, J. Nowotny (Ed.) Ceramic interfaces properties and applications, IOM Communications, cop., London 1998. 